\title{
ON CONTINUA WHOSE LINKS ARE NON-INTERSECTING
}

GAIL S. YOUNG, JR.

There are many examples known of compact continua no two of whose simple links intersect, even though uncountably many of the links are nondegenerate. On page 346 of his Foundations of point set theory $[1],{ }^{1} \mathrm{R}$. L. Moore has given an example of a compact continuum which is the sum of an uncountable collection of nondegenerate simple links, only a countable number of which intersect. In view of these results it seems natural to inquire whether there is a compact continuum which is the sum of an uncountable collection of mutually exclusive nondegenerate simple links. Theorem 1 gives a negative answer. If we do not require that the continuum be compact, but do require that it be a separable locally connected Moore space, ${ }^{2}$ then Theorem 7 shows that the answer is again negative.

References to theorem and page numbers refer to Moore's book, to which the reader is directed for definitions not given here.

THEOREM $1 .^{3}$ If $M$ is a compact metric continuum which is not a simple link of itself and no two of its simple links intersect, then uncountably many are degenerate, and the set which is the sum of the nondegenerate simple links of $M$ is the sum of a countable number of closed point sets. ${ }^{4}$

Lemma. Under the hypothesis of Theorem 1, the simple links of $M$ form an upper semi-continuous collection, $G$, filling up $M$ such that $G$ is an acyclic continuous curve every interval of which contains a degenerate element of $G$.

Proof. By Theorem 93, p. 67, each simple link of $M$ is a continuum. Let $P$ be the sequential limiting point of a sequence of points of distinct links $L_{1}, L_{2}, L_{3}, \cdots$ of $M$. Let $Q$ be any point of the limiting set of $L_{1}, L_{2}, L_{3}, \cdots$. Clearly no point separates $P$ from $Q$

Presented to the Society, April 29, 1944; received by the editors February 23, 1944.

${ }_{1}$ Numbers in brackets refer to the Bibliography at the end of the paper.

${ }^{2} \mathrm{~A}$ space is said to be a Moore space if it satisfies Axiom $\mathbf{0}$ and the first three parts of Axiom 1 of Moore's book.

${ }^{3}$ In the terminology of transformations, Theorem 1 is equivalent to the following: Let $f$ be a monotone mapping of the compact continuum $M$ into a dendrite $N$. If for each point $X$ of $N$ no point of $M$ separates any two points of $f^{-1}(X)$ and for each two points $X$ and $Y$ of $N$ some point of $M$ separates $f^{-1}(X)$ from $f^{-1}(Y)$, then for uncountably many points $X$ of $N$ the set $f^{-1}(X)$ is degenerate.

${ }^{4}$ The proof of this theorem was altered after refereeing. 
in $M$, and since by Theorem 103, p. 71, no point of $M$ is an improper point of $M$, it follows that the collection $G$ is upper semi-continuous. By Theorem 23(c), p. 344, $G$ is a dendrite with respect to its elements. Suppose that the interval $A B$ of $G$ contains no degenerate element. Then the set, $K$, of all points each of which separates $A$ from $B$ in $M$ is countable, for no link can contain more than two points of $K$, and by Theorem 24(a), p. 350, no more than a countable number of nondegenerate links can contain cut points of $M$. However, the collection, $T$, of all simple links of $M$ in $A B$ that intersect $K$ is dense in $A B$. From Theorem 24, p. 348, we have that $G$ contains an uncountable continuous subcollection, $C$, dense in $A B \cdot{ }^{5}$ Let $P$ be a point of $K$ contained in a link $L$, and let $M^{\prime}$ denote the subcontinuum of $M$ consisting of the sum of the elements of $A B$. Then $M^{\prime}-P$ is the sum of two mutually separated sets, $H_{1}$ and $H_{2}$, containing $A$ and $B$ respectively; and since $L$ separates $A B$ into two connected sets of elements of $G, M^{\prime}-P=L_{1}+L_{2}+(L-P)$, where $L_{1}$ and $L_{2}$ are mutually separated connected sets not intersecting $L$. If $L-P$ contains limit points of both $L_{1}$ and $L_{2}$, then it intersects both $K_{1}$ and $K_{2}$, which contradicts Theorem 98, p. 70 . Hence $L-P$ and $H_{1}$, say, are mutually separated. But since $C$ is dense in $A B$ it follows that there is a sequence $L_{1}, L_{2}, L_{3}, \cdots$ of elements of $C$ whose limiting set (in $M$ ) is $P$. Since $G$ is upper semi-continuous, as $n \rightarrow \infty$, diam $L_{n} \rightarrow 0$. Let $H$ be an element of $C$, and let $d$ be its diameter. Every open segment of $A B$ containing $H$ also contains elements of $T$, and therefore contains elements of $C$ of diameter less than $d / 2$, which contradicts the continuity of $C$. Hence $A B$ contains a degenerate link of $M$.

Proof of Theorem 1. By the lemma and Theorem 5.1, p. 131, of [4], the collection $G^{\prime}$ of all nondegenerate elements of $G$ is semiclosed, and each element of $G^{\prime}$ is a component of $G^{\prime *}$. By Theorem 5.41, p. 132 , of $[4], G^{*}$ is the sum of a countable number of closed point sets. If the degenerate elements of $G$ were countable in number, the decomposition space $G$ would be the sum of a countable number of closed sets, no one of which contains a domain, which would contradict Theorem 15, p. 11.

A well known example due to Anna M. Mullikin [2], and described on p. 366 of Moore's book, of a connected set in the plane which is the sum of a countable number of mutually exclusive arcs, can be modified by replacing each arc by a simple closed curve to show that Theorem 1 is not true if the hypothesis of compactness is replaced by separability, even if each link is a compact continuum.

Another argument for Theorem 1 could be given by proving that

5 Theorem 24 does not state that $C$ is dense in $A B$, but this readily follows here. 
under the hypothesis of Theorem 1 every simple link of $M$ is a set $M(P) .{ }^{6}$ Then Theorem 1 would follow from Theorem 2, which has some interest, due to the connection between dendratomic subsets, simple links, and sets $M(P)$ shown by Moore in chap. 5 of his book.

TheOREM 2. If the compact metric continuum $M$ is not an $M(P)$, then uncountably many sets $M(P)$ of $M$ are degenerate.

PRoof. By Theorem 23(a), p. 343, the collection, $G$, of all sets $M(P)$ of $M$ is upper semi-continuous. By Theorem 24(a), p. 350, no more than a countable number of nondegenerate elements of $G$ contain cut points of $M$. By hypothesis $M$ has uncountably many cut points. If the theorem were false some element of $G$ would contain uncountably many cut points of $M$, which contradicts Theorem 23(b), p. 344.

Since every simple link is a subset of some set $M(P)$, this theorem says that if the simple links are allowed to intersect in certain groups so that no two points in any group are separated by more than a countable number of points, but so that any two points in different groups are separated by uncountably many points, then the conclusion of Theorem 1 still holds.

Locally connected continua. If to the hypothesis of Theorem 1 we add the condition that $M$ be locally connected, our theorem becomes trivial, since the nondegenerate simple links of a compact continuous curve are its true cyclic elements, and there are only a countable number of these. If we also remove the condition of compactness, then the problem becomes more interesting, and indeed the theorem would not be true without the assumption of separability. However, if $M$ were assumed to be locally connected, separable, and complete, the theory of almost cyclic elements developed by F. B. Jones [3] would apply and our theorem could readily be proved, in virtue of Theorem 19 of his paper. Failure to assume completeness creates more difficulties, since arcwise connectivity would not be available. The theorem remains true, however, and the proof is not difficult. We prove first several theorems concerning simple links.

In the next four theorems, $M$ will denote a locally connected, connected Moore space.

TheOREM 3. ${ }^{7}$ If $L$ is a simple link of $M$, and $R$ is a connected domain intersecting $L$, then $L \cdot R$ is connected. Hence $L$ is connected and locally connected.

- If $P$ is a point of $M$, by $M(P)$ is meant the set of all points of $M$ which are not separated from $P$ by each of an uncountable set of points.

${ }^{7}$ Cf. Theorem 66, p. 147, of Moore, and Theorems 8 and 20 of Jones' paper. 
Proof. Suppose that $L \cdot R$ is the sum of two mutually separated sets, $H$ and $K$. Some component, $C$, of $R-R \cdot L$ has a limit point, $P$, in $H$, and a limit point, $Q$, in $K$, as may be seen with the use of Theorem 2(b), p. 91. Let $X$ be any point of $C$. No point of $L$ separates $P$ or $Q$ from $X$ in $R$. But by Theorem 12, p. 97,8 some point $Y$ separates $X$ from $P+Q$ in $R$ and is not itself separated from $P+Q$ by any point. Let $Z$ be a proper point of $M$ belonging to $L$. Some point, $W$, separates $Y$ from $Z$ in $M$, and $W$ can be neither $P$ nor $Q$. But then $W$ cannot belong to $L$, and hence separates $P+Q$ from $Y$, which is impossible.

If $R$ is taken to be $M$, it follows that $L$ is connected.

TheOREM 4. ${ }^{\circ}$ If $M$ is the sum of its.simple links, and no two simple links of $M$ intersect, then every improper point belongs to a nondegenerate continuum of improper points, no two lying in the same simple link.

Proof. Let $X$ be an improper point of $M$. Let $H$ be a point set containing $X$ such that no two points of $H$ belong to the same simple link of $M$ or are separated by any point, and which is maximal with respect to this property. Since the simple links of $M$ are mutually exclusive, $H$ is nondegenerate. Also, every point of $H$ is an improper point. Further, $H$ is closed, for if the point $Y$ of $M-H$ is a limit point of $H$, then no point separates $Y$ from any point of $H$. Hence $Y$ belongs to the same simple link as some point $Z$ of $H$. But then $Z$ separates $Y$ from $H-Z$, which is impossible.

Suppose that $H$ is the sum of two mutually exclusive closed sets, $H_{1}$ and $H_{2}$. Paralleling the argument of Theorem 3, we show the existence of a point $Q$ of $M-H$ and two points, $P_{1}$ and $P_{2}$, belonging to $H_{1}$ and $H_{2}$ respectively, such that no point separates $Q$ from both $P_{1}$ and $P_{2}$. Hence no point separates $Q$ from any point of $H$. Further, $Q$ is not in the same simple link as any point of $H$. Therefore $Q$ belongs to $H$, which is a contradiction, and completes our proof.

THEOREM 5. If $M$ is the sum of its simple links and is not a simple link of itself, and the simple links of $M$ are non-intersecting, they form an uncountable collection.

Proof. Suppose that the theorem is false. Then every point of $M$ is a proper point of $M$, for if $P$ were an improper point of $M$, by Theorem 4, $P$ would lie in a nondegenerate continuum $H$ of points, no two of which lie in the same simple link of $M$. But $H$ must be

${ }^{8}$ See remarks on p. 465 of Moore concerning this theorem.

- This theorem should be contrasted with Theorem 99, p. 71. 
countable, and no nondegenerate countable set is connected..$^{10}$ Hence, if $A$ and $B$ belong to distinct simple links of $M$, the set $I(A, B)$ of all points separating $A$ from $B$ is non-empty, and indeed any two points of $I(A, B)+A+B$ belonging to distinct links are separated by some point. Since no link can contain more than two points separating $A$ from $B, I(A, B)$ is countable. It is then easy to construct two sequences of points, $P_{1}, P_{2}, P_{3}, \cdots$ and $Q_{1}, Q_{2}, Q_{3}, \ldots$ such that (1) for each positive integer $i, P_{i+1}$ precedes $P_{i}, Q_{i+1}$ follows $Q_{i}$, and $P_{i}$ follows $Q_{i}$ in the order from $A$ to $B$ in $I(A, B)$; and (2) for any point $X$ of $I(A, B)$ there is an $i$ such that $X$ is not between $P_{i}$ and $Q_{i}$. But $P_{1}+P_{2}+P_{3}+\cdots$ cannot then have a limit point in $I(A, B)$, which contradicts Theorem 4.2 , p. 51 , of [4], the proof of which is valid in this space.

The example given following Theorem 1 shows that this theorem is not true without local connectivity.

The condition that $M$ is the sum of its simple links in the hypothesis of this theorem is necessary, for there exist non-compact continuous curves which do not contain any simple links whatsoever. We give a rough description of one such: Jones has given on p. 781 of [3] an example of a space, $H_{1}$, containing a simple closed curve, $C$, each of whose points is an improper point, and each of whose simple links is a simple closed curve, $C_{1, \alpha}$, having only a point in common with $C$. By applying Jones' construction to each set $C_{1, \alpha}$, we can construct a space $H_{2}$ in which every point of $H_{1}$ is an improper point, and whose simple links are simple closed curves, $C_{2, \alpha}$, having only one point in common with $H_{1}$. Repeating Jones' construction again on the sets $C_{2, \alpha}$, we obtain a space $H_{3}$ in which every point of $H_{2}$ is an improper point. Continue this process indefinitely. The space $H$ which is the limit of $H_{1}, H_{2}, H_{3}, \cdots$ is such that every point is an improper point, and hence $H$ has no simple links at all.

THeOREM 6. If $M$ is separable, the nondegenerate simple links of $M$ orm a countable collection, and each nondegenerate link is separable.

Proof. By hypothesis, $M$ contains a countable dense subset, $H$. By Theorem 12, p. 97, each nondegenerate simple link contains a cut point of $M$. Suppose that the collection, $G$, of all nondegenerate simple links of $M$ is uncountable. Either (1) there is a point, $P$, common to uncountably many elements of $G$, or (2) with Theorem 72 , p. 50 , and Theorem 68, p. 47 , there can be shown to exist an uncountable subcollection, $G^{\prime}$, of $G$, two points, $A$ and $B$, and a set, $K$, such

${ }^{10}$ Since a countable Moore space is completely separable, and hence metric, it cannot be connected, if it is nondegenerate. 
that each element of $G^{\prime}$ contains one and only one point of $K$, and such that each point of $K$ separates $A$ from $B$, and belongs to an element of $G^{\prime}$. In the last case, each element, $L$, of $G^{\prime}$ is intersected by a connected domain, $D_{L}$, whose closure does not intersect $I(A, B)+A+B$ (which by Theorem 4.2 , p. 51 , of [4] is closed and compact). There is a point, $X$, of $H$ which is common to uncountably many of the domains $D_{L}$. Let $G^{\prime \prime}$ denote the collection of all links, $L$, of $G^{\prime}$ such that $D_{L}$ contains $X$, and let $K^{\prime}$ denote the set of all points of $K$ intersecting links of $G^{\prime \prime}$. Some point, $Y$, is a point of condensation of $K^{\prime}$, and there is a connected domain, $D$, containing $Y$, such that for uncountably many elements $L$ of $G^{\prime \prime}$, $D_{L} \cdot D=0$. But then no point separates a point of an element of $G^{\prime \prime}$ from any point of another element of $G^{\prime \prime}$, which is impossible. A similar argument shows that the first case is likewise impossible, $P$ taking the role of $Y$.

We show that each simple link is separable. Let $L$ be any nondegenerate link of $M$, and let $H$ be a countable dense subset of $M$. For each point $X$ of $H-H \cdot M$, there is a point, $P_{X}$, of $L$ which separates $X$ from some point of $L$. The set, $H^{\prime}$, consisting of $H \cdot L$ and all points $P_{X}$ is countable. If $D$ is a connected domain intersecting $L$, and $Y$ is a point of $H \cdot D$, either $Y$ is in $L$ or $P_{Y}$ is in $D$. In either case, $D$ intersects $H^{\prime}$. Hence $H^{\prime}$ is a countable dense subset of $L$.

The last part of the theorem is false without local connectivity.

THEOREM 7. If $M$ is separable, and is not a simple link of itself, but is the sum of its simple links, and no two links of $M$ intersect, then uncountably many are degenerate.

PROoF. This follows immediately from Theorems 5 and 6 .

The example of Jones referred to following Theorem 4 shows that the condition of separability in Theorem 7 cannot be removed. A simple modification of this example gives a similar example where in addition the almost cyclic elements are mutually exclusive.

\section{BIBLIOGRAPHY}

1. R. L. Moore, Foundations of point set theory, Amer. Math. Soc. Colloquium Publications, vol. 13.

2. Anna M. Mullikin, Certain theorems relating to plane connected point sets, Trans. Amer. Math. Soc. vol. 24 (1922) pp. 144-162.

3. F. B. Jones, Almost cyclic elements and simple links of a continuous curve, Bull. Amer. Math. Soc. vol. 46 (1940) pp. 775-783.

4. G. T. Whyburn, Analytic topology, Amer. Math. Soc. Colloquium Publications, vol. 28.

Purdue University 